\title{
Assessment on the effect of pollution abatement on environmental efficiency with Markov chain Monte Carlo simulation
}

\author{
A. Ding ${ }^{1} \&$ S. Managi ${ }^{2}$ \\ ${ }^{1}$ Japan Society for Promotion of Science, Japan \\ ${ }^{2}$ Faculty of Business Administration, Yokohama National University, \\ Japan
}

\begin{abstract}
Both environmental economists and policy makers have shown a great deal of interest in the effect of pollution abatement on environmental efficiency. In line with the modern resources available, however, no contribution is brought to the environmental economics field with the Markov chain Monte Carlo (MCMC) application, which enables simulation from a distribution of a Markov chain and simulating from the chain until it approaches equilibrium. The probability density functions gained prominence with the advantages over classical statistical methods in its simultaneous inference and incorporation of any prior information on all model parameters. This paper concentrated on this point with the application of MCMC to the database of China, the largest developing country with rapid economic growth and serious environmental pollution in recent years. The variables cover the economic output and pollution abatement cost from the year 1992 to 2003 . We test the causal direction between pollution abatement cost and environmental efficiency with MCMC simulation. We found that the pollution abatement cost causes an increase in environmental efficiency through the algorithm application, which makes it conceivable that the environmental policy makers should make more substantial measures to reduce pollution in the near future.
\end{abstract}

Keywords: environmental efficiency, pollution abatement, Markov chain Monte Carlo, China, environmental productivity, environmental performance. 


\section{Introduction}

Society faces important tradeoffs between economic output and environmental policy. Both environmental economists and public policy makers in developed countries and developing countries alike have shown a great deal of interest in the effect of pollution abatement on environmental efficiency. In line with the modern resources available nowadays, a large amount of recent research was produced but no contribution to the environmental economics field with the Markov chain Monte Carlo (MCMC) application was brought. MCMC techniques enable simulation from a distribution of a Markov chain and simulating from the chain until it approaches equilibrium.

This simulation approach of probability density functions gained prominence by the practice of Bayesian statistics, which owns the advantages over classical statistical methods in its simultaneous inference and incorporation of any prior information on all model parameters. The work we performed in this paper concentrated on this point. In this paper, we apply Bayesian approach via MCMC simulation to the database of China, the largest developing country. China is the World's 3rd largest consumer of coal and oil, but much of its energy producing and using equipment is both inefficient and highly polluting. As a result, China experiences severe urban air pollution that has a significant impact throughout the region. It is also the World's second-largest source of greenhouse gas (GHG) emissions. Improving energy efficiency and accelerating the development and application of new and renewable energy and clean coal technologies are therefore very urgent sustainable development and environmental priorities. Public policy makers in China are facing the tradeoffs between economic growth and environmental protection. Growth of total factor productivity plays an important role in GDP growth in China.

China has achieved rapid economic growth in recent decades, however, growth is also seriously damaging the natural resource base and generating major environmental liabilities and caused serious environmental problems including land degradation, deteriorating water quality and water scarcity, severe air pollution and declining natural forest cover. There is a blossoming growth of literature, mainly theoretical, on the effects of pollution abatement on environmental efficiency. Two possibilities are concerned as encourage or discourage. (Jaffe et al. [1])

It is important to understand the interaction between productivity change and environmental policies, which influence the compliance costs. In the long run, the most important single criterion on which to judge environmental policies might be the extent to which they spur new technology toward the efficient conservation of environmental quality (Kneese et al. [2]).

In our study, the variable of the sample dataset covers the economic output and pollution abatement cost. The costs of pollution abatement technologies, which are important determinants of the environmental compliance cost, are also influenced by productivity (Jaffe et al. [1]).

Whether pollution abatement technologies are utilized more efficiently is crucial for the sustainable development. The principal focus of this paper is to 
measure the effect of pollution abatement on environmental efficiency using unique province-level secondary industry data over the period 1992-2003. The pollution variables include the wastewater discharge, waste gas emission, solid waste generation, SO2, dust, soot, $\mathrm{COD}$, lead and six chromium. The capital intensity (Capital) is analyzed since it might affect the productivity (Kagawa [3]).

In this paper, we test the causal direction between pollution abatement cost and environmental efficiency with the application of MCMC simulation. In contrast with the models of earlier studies, our model was able to make a causality test based on limited data set. We found that the pollution abatement cost cause increase in environmental efficiency through the algorithm application, which is conceivable that the environmental policy makers should make more substantial measures to reduce pollution in the near future.

In our knowledge, up to date no previous studies apply the MCMC method in environmental economics literature. In this study, we apply a Bayesian analysis based on MCMC methods in order to analyze the relationship between environmental efficiency and pollution abatement. The implementation of MCMC techniques is connected with further advantages as discussed below. Statistical inferences on states variables and parameters are easily performed via Bayesian MCMC methods. The flexibility of MCMC methods allows us to analyze a wide range of models with non-linear relationships in the dynamic and observation equations, and non-Gaussian error structure as well. The Bayesian paradigm is efficient for deriving quantitative diagnostics on a probability-based rationale. Outputs of the technique mainly consist of the joint posterior distribution of all the model parameters. Incorporating the likelihood above and the priors, using Bayesian rule, leads to the conditional posterior distributions for the parameter groupings used in the MCMC sampling scheme.

The use of MCMC is superior to other methods for analysis in limited data set. The remaining paper is planned as follows: Section 2 discusses China's environmental issues and the theoretical model of Markov Chain Monte Carlo. Section 3 describes the empirical model and the data used in estimation. Section 4 discusses the results while Section 5 provides conclusions.

\section{Background}

\subsection{China's environmental issues}

The environment has emerged as a leading concern in China. How degraded China's environment has become is a hot topic in current international environmental research field, and that further damage could hobble the economy. China began to implement environmental policies since the late 1970s with the issuing of Environmental Protection Law in 1979 responding to air pollution, water pollution, and solid waste disposal. The development of environmental policies also coincided with and was influenced by the economic reforms of the 1980s and the increased opening to international market. 
China has unveiled ambitious policies to promote eco-efficiency, created a "circular economy" based on sustainability and recycling, and begun to enact a climate change policy. The investment into and funding of science and innovation projects in China is growing rapidly, and its impact on the international community is also significant. Since 1999, China's spending on research and development (R\&D) has increased by more than 20 per cent a year. It has replaced Japan to become the world's highest spender on R\&D after the US.

The pollution levy system is the most long-standing component of China's regulatory structure for controlling pollution emissions and discharges. Chinese government establishes the Ministry of Environment and the Ministry of Energy with stronger regulatory capacity to achieve the balance of economic, environmental and social considerations, developing more environmentally friendly forms of energy production. China is the World's 3rd largest consumer of coal and oil, but much of its energy producing and using equipment is both inefficient and highly polluting. As a result, China experiences severe urban air pollution that has a significant impact throughout the region. It is also the World's second-largest source of greenhouse gas (GHG) emissions.

Improving energy efficiency and accelerating the development and application of new and renewable energy and clean coal technologies are therefore very urgent sustainable development and environmental priorities. Hereby we'd like to definite the eco-efficiency, the World Business Council for Sustainable Development describes eco-efficiency as "being achieved by the delivery of competitively priced goods and services that satisfy human needs and bring/improve quality of life, while progressively reducing ecological impacts and resource intensity throughout the life cycle to a level at least in line with the Earth's estimated carrying capacity" (Ehrenfeld [4]). As for the measures of relevant assessment in the literature, "pollution performance index" is employed given by the ratio between actual pollution levels (Cormier et al. [5]). The pollution standard is set in the Environment Administration field (Martin et al. [6]). A "pollutant intensity index" of a given plant based on pollutant risk and manufacturing activity is computed (Beede et al. [7]). Jaggi and Freedman [8] use a more elaborate indicator through computation between pollution performance and economic and market performance. Reviewing the above literature, we will assess the pollution abatement and the eco-efficiency (i.e. environmental efficiency).

\subsection{Markov chain Monte Carlo methods}

MCMC methods are a class of algorithms for sampling from probability distributions based on constructing a Markov chain that has the desired distribution as its stationary distribution. In very broad terms, Markov chains are processes describing trajectories where successive quantities are described probabilistically according to the value of their immediate predecessors.

A good chain will have rapid mixing - the stationary distribution is reached quickly starting from an arbitrary position-described further under Markov chain mixing time. MCMC methods have been applied in economic and social issues because of the simulation of Bayesian posterior density functions. The 
reason of employing Bayesian inference via MCMC in this paper is to overcome the drawbacks of maximum likelihood estimation (MLE).

In this paper, Metropolis-Hastings algorithm is applied to generate a random walk using a proposal density and a method for rejecting proposed moves. The Bayesian approach and its application via MCMC methods have several advantages. First, the subject-specific treatment distributions can be easily computed by simulating the latent variables (Chib and Jeliazkov [9]). Second, we do not have to apply numerical integration methods to evaluate the likelihood function of our model. This is particularly important when more flexible distributions than the normal are assumed for the error terms. Thirdly, it allows us to conduct exact small sample inference.

\section{Estimation}

We use economy-wide province level data tracked from 1992 to 2003 (See the detailed data description in the Appendix). MCMC method is used in the given situation. The main concern is efficient computation. Efficiency can be measured by the ease with which a simulated sample is obtained (Chen et al. [10]).

We make our inference on the base of a large sample of draws from the posterior distribution. Designing a Markov chain with a transition kernel generates the sample. The kernel has an invariant measure equal to the posterior distribution. We referred to this as a sequential sampling proposal process. We now describe a more general Metropolis-Hastings update where the proposal process is a truncated version of the same sequential sampling scheme.

The likelihood function $p(R \perp \Theta)$ is given by

$$
p\left(R^{2, n} \perp \Theta\right)=\prod_{t=2}^{n}\left\{\sum_{j=1}^{2} \frac{\Gamma((v+1) / 2}{\Gamma(v / 2) \sqrt{(v-2) \pi}} \frac{1}{\sqrt{h t}}\left[1+\frac{\left(R_{t}-\mu_{t}\right)^{2}}{(v-2) h t}\right]^{-(v+1) / 2} I_{j t}\right\},
$$

where $p$ is the posterior distribution, $R$ is the risk of posterior distribution, $\Theta$ is the Bayesian estimate, $I_{j t}$ is the indicator variable, $\Gamma(\cdot)$ is the Gamma function and $v$ is the degrees of freedom.

We set prior distributions on all model parameters following;

$$
\begin{array}{r}
p(\Theta) \propto I\left(\alpha_{0}^{(1)}>0, \alpha_{1}^{(1)}+\beta_{1}^{(1)}<1\right) I\left(\alpha_{0}^{(2)}>0, \alpha_{1}^{(2)}+\beta_{1}^{(2)}<1\right) \\
I(q 1<r k<q 3), I(d \in 1,2,3), I(t \in[0,0.25]),
\end{array}
$$

where $I(\cdot)$ is an indicator function, $q_{1}$ and $q_{3}$ are the first and third quantities, respectively, of the required threshold variable. In threshold modeling, we set a minimum amount of observations in each regime, so there is sufficient sample size to generate meaningful inference results, driving our prior choice for $r_{k}$. 


\section{Results and discussion}

This procedure produces a set of estimates that converges in the limit to the joint posterior distribution of the parameters (Gelfand et al. [11]). We ran the MCMC algorithm for 5,000 iterations with a burn-in phase of 10,000 iterations. The posterior standard deviation is used as a measure of our uncertainty about these point estimates. We do not have the posterior directly, however, calculating their sample analogues can closely approximate these quantities.

Table 1: Empirical results for the effect of pollution abatement on environmental efficiency.

\begin{tabular}{|l|c|c|c|}
\hline & Posterior means & $\begin{array}{c}\text { Posterior } \\
\text { deviations }\end{array}$ & Standard errors \\
\hline 1992 & 340.32 & 0.72 & 0.01 \\
\hline 1993 & 320.34 & 0.95 & 0.04 \\
\hline 1994 & 200.21 & 0.87 & 0.02 \\
\hline 1995 & 280.98 & 0.99 & 0.02 \\
\hline 1996 & 200.72 & 0.76 & 0.02 \\
\hline 1997 & 332.01 & 0.89 & 0.01 \\
\hline 1998 & 340.25 & 0.91 & 0.02 \\
\hline 1999 & 398.32 & 0.99 & 0.05 \\
\hline 2000 & 400.62 & 0.43 & 0.02 \\
\hline 2001 & 430.02 & 0.45 & 0.02 \\
\hline 2002 & 350.23 & 0.78 & 0.05 \\
\hline 2003 & 290.98 & 0.76 & 0.04 \\
\hline
\end{tabular}

Table 1 shows the results of hypothesis test of the non-causality on abatements and environmental efficiency regimes. The posterior means were obtained from the marginal posterior densities. The resulting posterior standard deviations are shown as well. For individual period, however, they express relatively larger posterior standard deviations corresponding to the practical intersection of observations.

In general, the posterior density based directly upon the likelihood function is much more tractable than the sampling distribution of a modal value of that function, which involves the maximization operator. In this study, the limited information analysis can now be carried out under a Bayesian approach leading to sharper finite sample results. We test the causal direction between pollution abatement cost and environmental efficiency with MCMC simulation. We found that the pollution abatement cost cause increase in environmental efficiency through the algorithm application, which is conceivable that the environmental policy makers should make more substantial measures to reduce pollution in the near future. Although China is the World's 3rd largest consumer of coal and oil, much of its energy producing and using equipment is both inefficient and highly polluting. Consequently, China experiences severe urban air pollution that has a significant impact throughout the region. China is the World's second-largest 
source of greenhouse gas (GHG) emissions, improving energy efficiency and accelerating the development and application of new and renewable energy and clean coal technologies are therefore very urgent sustainable development and environmental priorities.

The costs of alternative production and pollution abatement technologies are important determinants of the environmental compliance cost (Managi et al. [12]). In the long run, the most important single criterion on which to judge environmental policies might be the extent to which they spur new technology toward the efficient conservation of environmental quality. During the past years, China has implemented series of environmental policies, such as the promotion of biomass energy development. These measures have got desirable effect in power production and environmental conservation (Ding and Toyoda [13]). In the meanwhile, the stringency of these regulations is increasing such as the investment in urban waste treatment and water conservation. Along with the enforcement of environmental laws, most national and private enterprises began to pay attention to the environmental managements. The new established Ministry of Environmental Protection and the local Bureaus of Environmental Protection issue and regulate enterprises on their environmental conservation activities.

We employ economic techniques and find that efficiency in Chinese environmental management is improving. Our results show that Chinese environmental efficiency (or productivity) is increasing while pollution abatement is improving, though the effect is not obvious in some period. Our result is consistent with the literature of this topic (Jefferson et al. [14]), the pollution abatement and control expenditure positively affects to environmental productivity and technological progress as expected, while a problem we have to admit is that the environmental management in China has not effectively enough regulated wastewater, air and solid waste pollutants emissions over our study periods.

\section{Conclusions}

Environmental problem has been a top global treat. China is facing daunting challenges to address environment pollution and ecosystem degradation with its remarkable economic growth, and its future for a sustainable economic development relies on efficiency improvement. Given concerns over the rapidly economic growth and severely environmental degradation in China, Our study features various pollutants of China in 31 provinces from 1992 to 2003 . We focus on the estimation of the dataset of China with a new method in Bayesian analysis via MCMC simulation.

An advantage of this method is that the normal proposal density for this case becomes adaptive since it depends on the values of the other parameters from the current and previous iterations. This semiautomatic updating feature makes the proposal density closer to the true conditional posterior, which may lead to a more efficient algorithm. We test the effect of pollution abatement on environmental efficiency with MCMC simulation. We found that the pollution 
abatement cost cause increase in environmental efficiency through the algorithm application, which is conceivable that the environmental policy makers should make more substantial measures to reduce pollution in the near future.

\section{Appendix}

\section{Data description and source}

Labor

Number of employees refers to the persons who are engaged in social working and receive remuneration payment or earn business income (unit of 104). Source: Comprehensive Statistical Data and Materials on 50 Years of New China.

\section{Capital}

The capital stock is estimated from annual gross regional investment and depreciation rate (unit of 108 yuan). Source: China Industrial Economy Statistical Yearbook, and China Statistical Yearbook.

Pollution abatement

Pollution abatement cost and expenditure associated with the fund actually used for the environmental pollution of wastewater, waste gas and solid waste (unit of 108 yuan). Source: China Environmental Statistical Data and Materials, and China Statistical Yearbook.

Water

Wastewater quantity measured as the weight of wastewater discharge (unit of 104 ton). Source: China Environmental Statistical Data and Materials. Gas

Waste gas quantity measured as the volume of waste gas emissions, which is not treated (unit of $108 \mathrm{~m} 3$ ). Source: China Environmental Statistical Data and Materials.

Solid

Solid wastes quantity measured as the discharge amount of solid wastes (unit of 104 ton). Source: China Environmental Statistical Data and Materials. Levy

Effective levy rates for excess wastewater, waste gas, and solid waste. Source:

The China Environment Yearbook, and China Environmental Statistical Data and Materials.

Regional Dummy:

Provinces: Anhui, Fujian, Gansu, Guangdong, Guizhou, Hainan, Hebei, Heilongjiang, Henan, Hubei, Hunan, Jiangsu, Jiangxi, Jilin, Liaoning, Qinghai, Shaanxi, Shandong, Shanxi, Sichuan, Yunnan, and Zhejiang.

Four municipalities: Beijing, Tianjin, Shanghai and Chongqing, administratively directly under the central government.

Five autonomous regions: the equivalent of provinces: Guangxi, Inner Mongolia, Ningxia, Tibet, and Xinjiang.

Note: Hong Kong and Macao are special administrative regions that are not included in statistics of the People's Republic of China. The data of Tibet is not completely available, so is excluded in the computation. 


\section{References}

[1] Jaffe, A.B., Newell R.G., \& Stavins, R. Technological Change and the Environment, Handbook of Environmental Economics, Karl-Göran Mäler Jeffrey Vincent, Amsterdam: North-Holland, Elsevier Science, pp.23$33,2003$.

[2] Kneese, A.V., \& Schultze C.L. Pollution, Prices and Public Policy, Brookings Institution, Washington, DC., pp.122-126, 1978.

[3] Kagawa Shigemi, How does Japanese Compliance with the Kyoto Protocol affect environmental productivity in China and Japan? Structural Change and Economics Dynamics, pp.174-176, 2008.

[4] Ehrenfeld, J.R., Eco-efficiency: philosophy, theory, and tools, Journal of Industrial Ecology 9, pp.6-8, 2005.

[5] Cormier D., M. Magnan \& B. Morard, The impact of corporate pollution on market valuation: some empirical evidence, Ecological Economics, 8, pp.135-155, 1993.

[6] Martin P., D. Wheeler, M. Hettige \& R. Stengren, The industrial pollution projection system: concept, initial development and critical assessment, World Bank Discussion paper, pp.67-87, 1991.

[7] Beede D.N., D.E. Bloom \& D. Wheeler, Measuring and explaining crossestablishment variation in the generation and management of industrial waste, World Bank Discussion paper, pp.33-56, 1993.

[8] Jaggi B. \& M. Freedman, An examination of the impact of pollution performance on economic and market performance: pulp and paper firms, Journal of Business Finance and Accounting, 19, pp.697-713, 1992.

[9] Chib, S. \& Jeliazkov, I., Marginal likelihood from the Metropolis-Hastings Output, Journal of the American Statistical Association 96,pp.270$281,2001$.

[10] Chen, M.H, Q.M. Shao \& J.G. Ibrahim Monte Carlo Methods in Bayesian Computation. Springer Verlag, New York, pp.239-345, 2000.

[11] Gelfand, Allan E. \& Adrian F.M. Smith, Sampling-based approaches to calculating marginal densities. Journal of the American Statistical Association 85:pp.398-409.1990.

[12] Managi, S. J.J. Opaluch, D. Jin, \& T.A. Grigalunas. Technological Change and Depletion in Offshore Oil and Gas, Journal of Environmental Economics and Management, 47 (2): pp388-409,2004.

[13] Ding, A., \& T. Toyoda, Biomass Energy Development in Heilongiiang Province of China, Journal of Japanese Agricultural Economics, pp89-98, 2005.

[14] Jefferson, G.H., Rawski, T.G., L. Wang \& Y. Zheng, Ownership, Productivity Change, and Financial Performance in Chinese Industry. Journal of Comparative Economics, 28 (4): pp.786-813, 2000. 